\title{
A method to construct a points system to predict cardiovascular disease considering repeated measures of risk factors.
}

Antonio Palazón-Bru, Julio A Carbayo-Herencia, Maria Isabel Vigo, Vicente F Gil-Guillén

Current predictive models for cardiovascular disease based on points systems use the baseline situation of the risk factors as independent variables. These models do not take into account the variability of the risk factors over time. Predictive models for other types of disease also exist that do consider the temporal variability of a single biological marker in addition to the baseline variables. However, due to their complexity these other models are not used in daily clinical practice. Bearing in mind the clinical relevance of these issues and that cardiovascular diseases are the leading cause of death worldwide we show the properties and viability of a new methodological alternative for constructing cardiovascular risk scores to make predictions of cardiovascular disease with repeated measures of the risk factors and retaining the simplicity of the points systems so often used in clinical practice (construction, statistical validation by simulation and explanation of potential utilization). We have also applied the system clinically upon a set of simulated data solely to help readers understand the procedure constructed. 


\section{AUTHOR COVER PAGE:}

2 Title: A method to construct a points system to predict cardiovascular disease considering

3 repeated measures of risk factors.

4 Authors: Antonio Palazón-Bru, $\mathrm{PhD}^{1}$, Julio Antonio Carbayo-Herencia, $\mathrm{MD}, \mathrm{PhD}^{2}$, Isabel Vigo-

5 Aguiar, $\mathrm{PhD}^{3}$, Vicente Francisco Gil-Guillén, $\mathrm{MD}, \mathrm{PhD}^{1}$.

6 Institutions:

7 1. Department of Clinical Medicine, Miguel Hernández University, San Juan de Alicante,

8 Alicante, Spain.

9 2. Chair of Cardiovascular Risk, San Antonio Catholic University, Murcia, Region of Murcia, 10 Spain.

11 3. Department of Applied Mathematics, University of Alicante, San Vicente del Raspeig, 12 Alicante, Spain.

13 Corresponding author: Prof. Antonio Palazón-Bru, PhD. Department of Clinical Medicine,

14 Miguel Hernández University, Carretera de Valencia - Alicante S/N, 03550, San Juan de

15 Alicante (Spain). Phone number: +34 965919449. Fax number: +34 965919450. E-mail:

16 antonio.pb23@gmail.com 


\section{ABSTRACT}

18 Current predictive models for cardiovascular disease based on points systems use the baseline

19 situation of the risk factors as independent variables. These models do not take into account the

20 variability of the risk factors over time. Predictive models for other types of disease also exist

21 that do consider the temporal variability of a single biological marker in addition to the baseline

22 variables. However, due to their complexity these other models are not used in daily clinical

23 practice. Bearing in mind the clinical relevance of these issues and that cardiovascular diseases

24 are the leading cause of death worldwide we show the properties and viability of a new

25 methodological alternative for constructing cardiovascular risk scores to make predictions of

26 cardiovascular disease with repeated measures of the risk factors and retaining the simplicity of

27 the points systems so often used in clinical practice (construction, statistical validation by

28 simulation and explanation of potential utilization). We have also applied the system clinically

29 upon a set of simulated data solely to help readers understand the procedure constructed. 
31 Given that cardiovascular diseases (CVD) are one of the main causes of death in the world

32 (WHO, 2014), prediction models are interesting in order to determine those risk factors that can be acted on to reduce the probability of CVD (Molinero, 2003). The simplest model to make predictions about a dichotomous event, such as CVD, is logistic regression (Hosmer \& Lemeshow, 2000). This model produces an equation which, once the values for the various risk factors are known, can be used to evaluate the likelihood of the appearance of disease. However, this sort of model fails to consider exposure time. This is precisely what is done in survival models, which analyse the time of occurrence of a particular event. Although the best known of these models is Cox (Hosmer \& Lemeshow, 2008), it is not the only alternative available. There exist other possible methods to analyse survival, called parametric models as they assume a concrete type of distribution, such as the Weibull model, used in the SCORE project (Conroy et al., 2003). Indeed, the Framingham study used both logistic regression models and survival models (parametric and non-parametric) (National Heart, Lung, and Blood Institute, 2015). developed that are also used in clinical practice, though to a lesser extent, such as the Reynolds risk score and the WHO/ISH score (Cooney, Dudina \& Graham, 2009). Common to all these is the making of predictions about CVD over a 10-year period, though they consider different outcomes (morbidity and mortality with coronary heart disorders, mortality from coronary heart disorders, cardiovascular morbidity and mortality, or just cardiovascular mortality) and use different mathematical models (Cox and Weibull). These models enable physicians to make long-term decisions for their patients. In addition, the clinical practice guidelines recommend using these predictive models to stratify the cardiovascular risk of patients. For example, in 
53 Europe, the European Guidelines on cardiovascular disease prevention in clinical practice

54 indicate "A risk estimation system such as SCORE can assist in making logical management

55 decisions, and may help to avoid both under-and overtreatment" (Perk et al., 2012). In other

56 words, clinicians follow the guidelines to improve the decision-making process in order to prevent CVD, and it is these very guidelines that indicate the use of these predictive models. Accordingly, these models are very relevant in daily clinical practice.

Given the complexity of these mathematical models an algorithm is used to enable the

60

clinician to understand them more easily, though precision is lost in the estimation of the probability of CVD (Sullivan, Massaro \& D'Agostino, 2004). To do this the mathematical models have been transformed into coloured risk tables that can be used systematically in clinical practice. However, these tables are based on models that manage clinical variables in the baseline situation of the patient (Conroy et al., 2003; National Heart, Lung, and Blood Institute, 2015), and do not therefore take into account the variability of the variables over time, as the biological parameters are being considered constant over the follow-up period when in fact they vary greatly and the physician can intervene using drugs to either reduce or increase their value (NCEP, 2002; American Diabetes Association , 2014; James et al., 2014; Stone et al., 2014).

Predictive models for survival in other diseases do consider the temporal variability of a single biological marker (as well as the baseline variables). These are known as Joint Models for Longitudinal and Time-to-Event Data and comprise two parts: 1) A mixed linear model to determine the path of a longitudinal parameter and 2) A survival model relating the baseline variables and the longitudinal parameter with the appearance of an event. These models can be used to make more precise predictions about the development of a disease (Rizopoulos, 2012). However, due to their complexity they are not used in general clinical practice. In addition, joint 
modelling when the survival part is formed by a linear function with multiple longitudinal parameters (usual modelling in traditional survival analysis in the health sciences) has only been examined theoretically and currently remains a complete computational challenge. This has resulted in the development of algorithms to make predictions, as in the univariate case (Rizopoulos, 2011).

Here we aim to show the viability and properties of a new methodological alternative for constructing cardiovascular risk scores (construction, statistical validation by simulation and potential utilization with the new theoretical model) dealing with the temporal variability of CVD risk factors. We also apply the model using a set of simulated data, with the sole purpose of helping readers understand how to apply it to a real data set with repeated measures of cardiovascular risk factors. In other words, the example given using simulated data is only to show how to apply the method proposed with a real data set having the characteristics given in this work; thus the scoring system given here has no value in clinical practice; what is of value is the way the system is constructed.

\section{MATERIALS AND METHODS}

The basic models used to develop the new method were the Cox model with time-dependent variables, points system in the Framingham Heart Study, Joint Models for Longitudinal and Time-to-Event Data, and predictions of the longitudinal biomarkers using these Joint Models.

\section{Cox model with time-dependent variables}

Let $T$ be a non-negative random variable denoting the observed failure time, which is the minimum value of the true event time $T^{*}$ and the censoring time $C$ (non-informative right 
97 censoring). In other words, $T=\min \left(T^{*}, C\right)$. In addition, we define $\delta$ as the event indicator,

98 which takes the value 1 if $T^{*} \leq C$ and 0 otherwise. On the other hand, let $\boldsymbol{W}$ be the vector of

99 baseline covariates and $\boldsymbol{Y}(t)$ the vector of time-dependent covariates, assuming a defined value

100 for $t \geq 0$. With these data, the Cox model with time-dependent variables takes the following

101 form (risk function):

102

$$
\mathrm{h}(t \mid \boldsymbol{w}, \boldsymbol{y}(t))=\mathrm{h}_{0}(t) \exp \left\{\boldsymbol{\gamma}^{T} \boldsymbol{w}+\boldsymbol{\alpha}^{T} \boldsymbol{y}(t)\right\}
$$

where $\mathrm{h}_{0}(t)$ is the baseline risk function, and $\boldsymbol{\gamma}$ and $\boldsymbol{\alpha}$ are the vectors of the regression

coefficients for the baseline and time-dependent covariates, respectively (Andersen \& Hill, 1982).

The estimation of the model parameters is based on the partial likelihood function (Andersen \& Hill, 1982). On the other hand, we have to corroborate whether the functional form of the covariates in the model is linear. This should be performed using graphical methods (Martingale residuals against the covariate of interest). Finally, we have to assess whether the model fits the data well, through the analysis of the Cox-Snell residuals (graphical test).

112 from the above expression. Furthermore, the model has to verify the following condition

113 (proportional hazard assumption):

$$
\log \left(\frac{\mathrm{h}(t \mid \boldsymbol{w})}{\mathrm{h}_{0}(t)}\right)=\boldsymbol{\gamma}^{T} \boldsymbol{w}
$$


116 We summarize the steps of the method developed by the Framingham Heart Study to adapt a

117 Cox regression model with $p$ covariates to risk charts (Sullivan, Massaro \& D'Agostino, 2004):

118 1) Estimate the parameters of the model: $\hat{\boldsymbol{\gamma}}$.

119 2) Organize the risk factors into categories and determine reference values:

120 a. Continuous risk factor (e.g., age): set up contiguous classes and determine reference values for 121 each. Example for age: 18-30 [24], 30-39 [34.5], 40-49 [44.5], 50-59 [54.5], 60-69 [64.5] and $122 \geq 70$ years [74.5]. In brackets is the reference value. The Framingham Heart Study researchers 123 recommend mid-points as acceptable reference values, and for the first and last class the mean 124 between the extreme value and $1^{\text {st }}$ (first class) or $99^{\text {th }}$ percentiles (last class).

125 b. Binary risk factors (e.g. gender, 0 for female and 1 for male): the reference value is again 126 either 0 or 1.

127 Let $W_{i j}$ denote the reference value for the category $j$ and the risk factor $i$, where $i=1, \ldots, p$ and $j$ $128=1, \ldots, c_{i}$ (total number of categories for the risk factor $i$ ).

129 3) Determine the referent risk factor profile: the base category will have 0 points in the scoring 130 system and it will be denoted as $W_{i R E F}, i=1, \ldots, p$.

131 4) Determine how far each category is from the base category in regression units: calculate $\hat{\gamma}_{i}$.

$132\left(W_{i j}-W_{i R E F}\right), i=1, \ldots, p$ and $j=1, \ldots, c_{i}$.

133 5) Set the fixed multiplier or constant $B$ : the number of regression units equivalent to 1 point in

134 the points system. The Framingham Heart Study generally uses the increase in risk associated 135 with a 5-year increase in age. 
136 6) Determine the number of points for each of the categories of each risk factor: the closest

137 integer number to $\hat{\gamma}_{i} \cdot\left(W_{i j}-W_{i R E F}\right) / B$.

138 7) Determine risks associated with point totals: $1-\hat{S}_{0}(t) \exp \left\{\sum_{i=1}^{p}\left(\hat{\gamma}_{i} \cdot W_{i R E F}\right)+B \cdot P o i n t s-\sum_{i=1}^{p} \hat{\gamma}_{i} \cdot \hat{\boldsymbol{w}}_{i}\right\}$,

139 where $\hat{S}_{0}(t)$ is calculated through the Kaplan-Meier estimator.

\section{Joint Models for Longitudinal and Time-to-Event Data}

141 Using the former notation, we have the random variables vector $\{T, \boldsymbol{W}, Y(T)\}$, where $Y(T)$ is only

142 a time-dependent variable (longitudinal outcome) which has its values defined intermittently for

$143 t$. In other words, for a subject $(i=1, \ldots, n), y(t)$ is only defined for $t_{i j}\left(j=1, \ldots, n_{i}\right), y_{i}\left(t_{i j}\right)$,

144 where $0 \leq t_{i 1} \leq t_{i 2} \leq \ldots \leq t_{i n_{i}}$. Now, we will denote as $m(t)$ the true and unobserved value of

145 the longitudinal outcome at time $t\left(m_{i}(t)\right.$ for the subject $\left.i\right)$. To assess the effect of $m(t)$ on the

146 event risk, a standard option is to adjust a Cox regression model with one time-dependent

147 covariate:

$$
\mathrm{h}(t \mid \mathcal{M}(t), \boldsymbol{w})=\mathrm{h}_{0}\left(t^{*}\right) \exp \left\{\boldsymbol{\gamma}^{T} \boldsymbol{w}+\alpha m(t)\right\}
$$

where $\mathrm{M}(t)$ for a subject $i$ is defined as $\mathrm{M}_{i}(t)=\left\{m_{i}(u) ; 0 \leq u<t\right\}$, which denotes the history of the true unobserved longitudinal process up to time $t$. The other parameters in the expression follow the structure of the Cox regression model with time-dependent variables (see former section). The baseline risk function can be unspecified or can be approximated with splines or step functions (Rizopoulos, 2012). 
155 However, in our sample we have $y(t)$; therefore we will estimate $m(t)$ using $y(t)$ through a

156 linear mixed effects model to describe the subject-specific longitudinal evolutions:

$$
\left\{\begin{array}{c}
y_{i}(t)=m_{i}(t)+\varepsilon_{i}(t) \\
m_{i}(t)=\boldsymbol{x}_{\boldsymbol{i}}^{T}(t) \boldsymbol{\beta}+\mathbf{z}_{\boldsymbol{i}}^{T}(t) \boldsymbol{b}_{\boldsymbol{i}} \\
\boldsymbol{b}_{\boldsymbol{i}} \sim N(\mathbf{0}, \boldsymbol{D}) \\
\varepsilon_{i}(t) \sim N\left(0, \sigma^{2}\right)
\end{array}\right.
$$

where $\boldsymbol{\beta}$ and $\boldsymbol{b}_{\boldsymbol{i}}$ denote the vectors of regression coefficients for the unknown fixed-effects parameters and the random effects respectively, $\boldsymbol{x}_{\boldsymbol{i}}(t)$ and $\boldsymbol{z}_{\boldsymbol{i}}(t)$ denote row vectors of the design matrices for the fixed and random effects respectively, and $\varepsilon_{i}(t)$ is the error term with variance $\sigma^{2}$. Finally, $\boldsymbol{b}_{\boldsymbol{i}}$ follows a normal distribution with mean $\mathbf{0}$ and covariance matrix $\boldsymbol{D}$, and independent of $\varepsilon_{i}(t)$ (Rizopoulos, 2012).

The estimation of the parameters of the joint models is based on a maximum likelihood approach that maximizes the log-likelihood function corresponding to the joint distribution of the time-to-event and longitudinal outcomes (Rizopoulos, 2012).

Regarding the assumptions of the model, we have to assess them for both submodels (longitudinal and survival) using the residual plots. For the longitudinal part, we will plot the subject-specific residuals versus the corresponding fitted values, the Q-Q plot of the subjectspecific residuals, and the marginal residuals versus the fitted values. On the other hand, for the survival part, we will plot the subject-specific fitted values for the longitudinal outcome versus the martingale residuals, and finally we will determine graphically whether the Cox-Snell residuals is a censored sample from a unit exponential distribution (Rizopoulos, 2012). 
173 Regarding, the last component (random effects part) of the joint model for which we have

174 indicated an assumption, other authors have showed that linear mixed-effects models are

175 relatively robust to misspecification of this distribution (Verbeke \& Lesaffre, 1997).

176 Predictions of the longitudinal biomarkers using these Joint Models for Longitudinal and Time-

177 to-Event Data

178 Let $\left\{t_{i}, \delta_{i}, w_{i}, y_{i}\left(t_{i j}\right), 0 \leq t_{i j} \leq t_{i}, j=1, \ldots, n_{i}\right\}, i=1, \ldots, n$ be a random sample of the random

179 variables vector $\{T, \Delta, \boldsymbol{W}, Y\}$, using the former notation. A joint model has been fitted using this

180 sample. Now, we are interested in predicting the expected value of the longitudinal outcome at

181 time $u>t$ for a new subject $i$ who has a history up to the time t of the observed longitudinal

182 marker $\mathcal{Y}_{i}(t)=\left\{y_{i}(s) ; 0 \leq s<t\right\}$ :

$$
\omega_{i}(u \mid t)=E_{Y}\left\{y_{i}(u) \mid t_{i}^{*}>t, \mathcal{Y}_{i}(t), \boldsymbol{w}_{\boldsymbol{i}} ; \boldsymbol{\theta}\right\}
$$

where $\boldsymbol{\theta}$ denotes the parameters' vector of the joint model (Rizopoulos, 2011).

Step 1: Draw $\boldsymbol{\theta}^{(l)} \sim \mathcal{N}(\hat{\boldsymbol{\theta}}, \widehat{\operatorname{var}}(\hat{\boldsymbol{\theta}}))$.

Step 2: Draw $\boldsymbol{b}_{\boldsymbol{i}}^{(l)} \sim\left\{\boldsymbol{b}_{\boldsymbol{i}} \mid t_{i}^{*}>t, \mathcal{Y}_{i}(t), \boldsymbol{w}_{\boldsymbol{i}} ; \boldsymbol{\theta}^{(l)}\right\}$

Step 3: Compute $\omega_{i}^{(l)}(u \mid t)=\boldsymbol{x}_{\boldsymbol{i}}^{T}(u) \boldsymbol{\beta}^{(l)}+\boldsymbol{z}_{\boldsymbol{i}}^{T}(u) \boldsymbol{b}_{\boldsymbol{i}}^{(l)}$ 
190 This scheme should be repeated $L$ times. The estimation of the parameter is the mean (or median)

191 of the calculated values $\left(\omega_{i}^{(l)}(u \mid t), l=1, \ldots, L\right)$ and the confidence interval is formed by the

192 percentiles (95\%: 2.5\% and 97.5\% percentiles) (Rizopoulos, 2011).

We highlight that these predictions have a dynamic nature, that is, as time progresses

194

195

196

197

198

199

200

201

202

203

204

205

206

207

208

209

210

\section{Construction}

We wish to determine the probability of having CVD with effect from a baseline situation $(t=0$

) up to a fixed point in time $(\tilde{t})$, given a series of risk factors measured at baseline and during this follow-up. To do this requires the following steps:

1) Adjust a Cox regression model with time-dependent variables. As we are unable to estimate a joint model with multiple longitudinal parameters (Rizopoulos, 2012), we use the classic extended Cox model (with no shared structure), which requires knowing the values of all the longitudinal parameters at any value of $t$. As this is not known because the parameters are recorded intermittently, we take the last value in time as a reference.

2) Use the procedure of the Framingham study to adapt the coefficients of the model obtained to a points system and determine the probabilities of CVD for each score up to the moment $\tilde{t}$. We then use these probabilities to construct risk groups that are easy for the clinician to understand (for example, in multiples of 5\%) (Sullivan, Massaro \& D'Agostino, 2004).

3) Adjust a joint model for longitudinal and time-to-event data for each longitudinal parameter recorded during the follow-up. This will also include all the baseline variables. These models are 
211 constructed to make predictions about the longitudinal parameters in new patients (statistical

212 validation by simulation and potential utilization).

213 Statistical validation by simulation

214 Once the points system has been constructed, we wish to see whether the model determines the 215 onset of CVD accurately in a different set of subjects (validation sample). In this validation

216 sample we know the longitudinal markers up to the point $t=0$ [record of cardiovascular risk

217 factors in the clinical history which were measured before the baseline situation $(t<0)]$ and the

218 value of the variables at baseline. With this information we determine the probability each

219 subject has of experiencing an event, and we then compare this with what actually occurred; i.e.,

220 determine whether the model is valid. To determine this validity we follow these steps:

221 1) Determine $L$ simulations of the longitudinal parameters at the time point $\tilde{t}$ using the models 222 mentioned in step 3) of construction, from the history $(t<0)$ and the baseline variables $(t=0)$

223 (Rizopoulos, 2011). We will use these simulated values to construct a distribution of the points

224 for each sample subject. Thus, each subject will have $L$ values for the points variable (evaluating

225 the points system using the simulated values and the baseline variables is sufficient), and for

226 each $l^{\text {th }}$ simulation each patient will have a points score. In other words, each simulation will

227 have a distribution of the points variable.

2) For each $l^{\text {th }}$ simulation adjust a classic Cox model (without time-dependent variables), using

229 just the score obtained as the only explanatory variable. Determine the Harrell's concordance statistic for each of these $L$ models. These values will give us a distribution of values for this

231 statistic, with which we calculate the mean (or the median) and the $2.5 \%$ and $97.5 \%$ percentiles

232 (Rizopoulos, 2011). This way we construct a confidence interval for this statistic, which will 
233 indicate the discriminating capacity of the points system to determine which patients will

234 develop CVD.

235 3) Calculate the median of the points distribution for each patient in the validation sample. Note

236 that we do not use the mean as it could contain decimals and this has no sense when applying the

237 scoring system. Using these medians, classify each patient in a risk group and compare the rate

238 of events predicted by the points system in each group to the actual observed rate. The test used

239 for this process will be Pearson $\chi^{2}$ test.

240 The concordance statistic used has been reported to have various limitations (Lloyd-

241 Jones, 2010). For example, it does not compare whether the estimated and observed risks are

242 similar in the subjects. Accordingly we have added the analysis of the differences between the

243 expected events and the observed events, which minimises this particular problem. In addition, it

244 is very sensitive to large hazard ratio values $(\geq 9)$. Nonetheless, we have to consider that as all the

245 variables are quantitative (not categorized), the hazard ratio values do not surpass this threshold.

246 Accordingly, the joint analysis of the concordance index of Harrell and the differences between

247 the expected and the observed events enables us to validate statistically by simulation of the

248 proposed model.

Explanation of potential utilization

250 Once the points system has been validated statistically the clinician can then apply the system to

251 determine the cardiovascular risk in a new patient, and take any necessary measures to reduce

252 this risk. The healthcare professional will already have historical information about the

253 longitudinal parameters $(t<0)$ and information about the baseline situation $(t=0)$ of the new

254 patient. The steps to be followed by the clinician are: 
255 1) Determine the value of each longitudinal parameter at the time $\tilde{t}$. To do this we apply the 256 models obtained in step 3) of construction to the history and the baseline situation of the new

257 patient, in order to determine $L$ simulations for each longitudinal parameter, similar to what was

258 done in the validation process. For each $l^{\text {th }}$ simulation we determine the score corresponding to

259 the profile of cardiovascular risk factors obtained (simulated and baseline information values).

260 This will give us a points distribution for the new patient.

261 2) Determine the median and the $2.5 \%$ and $97.5 \%$ percentiles of the points vector constructed

262 above. The median will be the estimation of the score for the new patient and the percentiles will

263 define the confidence interval (Rizopoulos, 2011). As each score has an associated risk, the

264 healthcare professional will be able to know the probability of CVD at time $\tilde{t}$, together with its

265 confidence interval. Finally, the clinician will know the values of the biological parameters at $\tilde{t}$

266 of the median of the points system. This way the clinician will be able to see which of these

267 parameters has a score above normal; i.e., see the possible areas of intervention to reduce the

268 cardiovascular risk.

269 3) The clinician now knows the cardiovascular risk and which parameters have a score above

270 normal, so he or she can then design the best intervention for that patient. This presents a

271 problem, as we need to know the value of each biological parameter at time $\tilde{t}$; i.e., the clinician

272 knows an approximation based on simulations constructed from the patient history but does not

273 know how the interventions will affect the cardiovascular risk.

From the previous step the clinician knows the parameters on which to act and the history

of these parameters as well as the baseline situation. From these measurements the clinician can

276 establish a realistic objective for the next patient visit at time $\tilde{t}(0<\tilde{t}<\tilde{t})$. The clinician now 
277 inserts the desired value of the biological parameter at $\tilde{t}$ and determines its value at time $\tilde{t}$; i.e.,

278 determine $L$ simulations for each cardiovascular risk factor using the previous models (step 3 of

279 construction), adding a new value to the history $(\tilde{\tilde{t}})$.

280 These calculations will give the benefit of the intervention (estimation [mean or median]

281 of the biological parameter at $\tilde{t}$ ) and the clinician will be able to see from the points system how

282 the patient's risk will be reduced.

\section{Simulation on a data set}

284 With the sole purpose of explaining how to use the method proposed here, we have simulated a

285 data set upon which to apply each of the steps described above. Note that we are in fact going to

286 simulate two data sets, one to construct the model and the other to validate it statistically via

287 simulation. So that both sets are biologically plausible we have used estimations obtained in the

288 Puras-GEVA cardiovascular study, which has been published in Medicine (Artigao-Ródenas et

289 al., 2015).

290

Our data sets will include the following biological parameters: age (years), systolic blood

291 pressure (SBP) (mmHg), HbAlc (\%), atherogenic index, gender (male or female) and smoking

292 (yes or no). Of these, the SBP, HbA1c and the atherogenic index will be present at baseline $(t$

$293=0)$ and in the follow-up for the construction sample $(t>0)$ or recorded in the clinical history

294 for the statistical validation sample via simulation $(t<0)$. The choice to include these variables

295 was based on the current cardiovascular risk scales (Conroy et al., 2003; National Heart, Lung,

296 and Blood Institute, 2015), except for HbA1c, which is used instead of a diagnosis of diabetes

297 mellitus in order to include another time-dependent parameter in the final model, in addition to 
which this way enables us to value the control of the diabetes mellitus (HbAlc $<6.5 \%)$ when preventing CVD.

For the main variable (time-to-CVD) we shall suppose that our cohort is used to predict CVD with a follow-up of 2 years. Note that the traditional cardiovascular risk scales use a time of 10 years (Conroy et al., 2003; National Heart, Lung, and Blood Institute, 2015). We have used this lower value because we are going to make predictions for the longitudinal parameters with effect from the baseline situation $(t=0)$ up to the prediction time and if we take a prediction value of 10 years the predictions for the longitudinal parameters will vary greatly and not allow us to make precise predictions about which patient will develop CVD, which would negate the usefulness of the method proposed here. Nevertheless, the fact that the predictions for the longitudinal parameters have a dynamic character (see Predictions of the longitudinal biomarkers using these Joint Models for Longitudinal and Time-to-Event Data) enables us to determine the risk at 2 years with greater precision whenever the patient attends the office of the healthcare professional. Note that the method proposed here has been developed for a theoretical time period $\tilde{t}$ but it can be applied for any time period. Nonetheless, generally speaking the longitudinal parameters would vary more over longer time periods, though this clearly depends on the nature of the data, both at the individual level and the population level (Rizopoulos, 2012).

The work used for our simulated data set developed and validated a predictive model of CVD (angina of any kind, myocardial infarction, stroke, peripheral arterial disease of the lower limbs, or death from CVD), to enable calculation of risk in the short, medium and long term (the risk associated with each score was calculated every 2 years up to a maximum of 14) in the general population (Artigao-Ródenas et al., 2015). Table 4 of this scoring system shows the 
321 importance of this question. For example, a patient with a score of 9 points has a probability of

322 CVD at 2 years of $0.67 \%$, whereas at 10 years this rises to 5.16\% (Artigao-Ródenas et al., 2015).

323 If we regularly calculate the 2-year risk of CVD for our patient and the score remains the same

324 then no new therapeutic action will be taken (risk $<1 \%$ ), whereas if we only calculate the risk

325 once every 10 years we will take aggressive therapeutic measures when the patient first attends

326 the office, as the score will correspond to a cut point defined as high in the SCORE project

$327(5 \% \rightarrow$ one in 20 patients) (Conroy et al., 2003). We see, then, that a regular short-term

328 prediction could lead to a change in the therapeutic decisions regarding prevention of CVD,

329 provided of course that the possibility exists of calculating the risk regularly. As the risk table

330 given in the Puras-GEVA study includes predictions for 4, 6, 8, 10, 12 and 14 years, we selected

331 the lowest cut point because if we had to make predictions for a longer time the dispersion could

332 have increased (Rizopoulos, 2012). This is why we chose this cut point of 2 years for the

333 simulation.

The longitudinal follow-up measurements (construction sample) assumed that the patient

335

336

337

338

339

340

341 attends the physician's office once every 3 months for measurements of SBP, HbA1c and the atherogenic index. This is done until the end of the follow-up for each patient. The statistical validation sample using simulation supposes that there is a certain probability of having records in the clinical history of all the longitudinal parameters every 3 months for 5 years retrospectively $(t<0)$. The probability is different for each of the visits and will depend on each patient. In other words, we will have intermittent measurements of all these parameters from $t=$ -5 years to $t=0$. 

variability in the cardiovascular risk factors. However, in Other S1 we can see that the models models would contain the constant with a very small random error. In other words using this prediction time makes sense.

We decided to use a simulated data set as we did not have available any data set with real data. This way of explaining a new method has already been used by others working with joint models, as the only objective of the simulated data set is to explain how to apply the new method (Faucett \& Thomas, 1996; Henderson, Diggle \& Dobson, 2000; Wang \& Taylor, 2001; Brown,

Ibrahim \& DeGruttola, 2005; Zeng \& Cai, 2005; Vonesh, Greene \& Schluchter, 2006; Rizopoulos \& Ghosh, 2011).

\section{RESULTS}

Given the amount and extension of the results these are given in detail in the supplementary material (Other S2 and Other S3). However, we have provided here the main results of our example. As before, the analysis was done with R 2.13.2 and IBM SPSS Statistics 19. 
362 The parameters of the Cox model with time-dependent variables are shown in Table 1, and its

363 adaptation to the points system with a prediction time of 2 years is reflected in Fig. 1. Table 2

364 shows the joint models for the longitudinal parameters. To avoid computational cost simple

365 models were used: 1) linear equation for the survival part with all the predictors included (age,

366 gender, smoking, and longitudinal marker) and 2) mixed linear model with polynomial degree 1

367 at $(1, t)$, in both the fixed and the random parts. The baseline risk function was defined 368 piecewise.

369

370

371

372

373

374

375

376

377

378

379

380

381

382

383

\section{Statistical validation by simulation}

The C-statistic was very satisfactory: 0.844 (95\% CI: 0.842-0.846). Comparison between expected and observed events in all the risk groups showed no significant differences (Fig. 2).

\section{Explanation of potential utilization}

A new patient arrives at our office with the following characteristics: male, 83 years old, nonsmoker, and taking pharmacological medication (one antihypertensive drug and one oral antidiabetic agent) and non-pharmacological measures (diet and exercise). His history of cardiovascular risk factors is available (Table 3).

Application of the new model gives a histogram of the cardiovascular risk score obtained for this patient (Fig. 3). This chart shows a high cardiovascular risk, as most of the simulations have around 16 points. The estimation of the score was 16 (95\% CI: 15-17). The median score corresponded to a SBP of $160 \mathrm{mmHg}, \mathrm{HbA} 1 \mathrm{c}$ of $5.0 \%$ and an atherogenic index of 6.76. Bearing in mind that the model contains factors upon which it is not possible to act (gender and age) that give the patient a minimum of 13 points, we should consider strategies to help the patient not to score in the other categories on the scale (Fig. 1). 
385 [pharmacological (add two antihypertensive drug $\rightarrow-20 \mathrm{mmHg}$; prescribe a statin $\rightarrow-40 \%$

386 atherogenic index) and non-pharmacological (reduce salt in the diet $\rightarrow-5 \mathrm{mmHg}$ )], his

387 longitudinal parameters after 3 months would be: SBP $120 \mathrm{mmHg}(145-2 \cdot 10-5=120$

$388 \mathrm{mmHg}$ ), atherogenic index $3.10(5.17-40 \%=3.10)$, and HbA1c 4.9\% (same value because no

389 intervention was done). Applying the model using the new information gives the cardiovascular

390 risk at 2 years (Fig. 4). The estimation of the score is 15 (95\% CI: 14-15) and the values that

391 provide a median score are: SBP $124 \mathrm{mmHg}$, atherogenic index 4.85, and HbA1c 5.0\%. Thus,

392 the risk is reduced, as now the patient has 15 points (Fig. 1 and S3 Other).

This paper describes a method to construct predictive models for CVD considering the variability of cardiovascular risk factors and at the same time having the simplicity of points systems, which are widely used in daily clinical practice worldwide (Conroy et al., 2003; Cooney, Dudina \& Graham, 2009; National Heart, Lung, and Blood Institute, 2015). the parameters controlling the risk factors, although a very positive aspect of these scales is that they take into account simplicity for immediate application by healthcare professionals, the persons who really have to apply these mathematical models (Conroy et al., 2003; Cooney, Dudina \& Graham, 2009; National Heart, Lung, and Blood Institute, 2015). The joint models currently used do take into account variability over time of a single longitudinal parameter (Rizopoulos, 2011), but their interpretation is not as easy as a points system and they cannot be 
406

407

408

409

410

411

412

413

414

415

416

417

418

419

420

421

422

423

424

425

426

427

428

used with various longitudinal parameters, a key question in the multifactorial aetiology of CVD.

We have attempted to fuse all these techniques into one single algorithm, retaining the virtues of each (relative risks model, scoring systems, dynamic predictions...).

Comparison between our proposed model and current cardiovascular risk scales is problematic. Our model is more suitable to make short-term predictions, though the more time that passes from the baseline situation $(t=0)$ when making a prediction, the variability of the predictions of the longitudinal parameters increases (Rizopoulos, 2012). This same situation can be found in other areas, such as the economy (stock exchange) or meteorology (weather forecast), though it obviously depends on the nature of the data being used, both at the individual level and the population level. This however does not weaken our model, since because the predictions for the longitudinal parameters are dynamic (Rizopoulos, 2011), any time that we update the clinical information about our patient the risk is immediately recalculated. This can be seen in the proposed example (Other S2 and Figs. 3 and 4), where when we introduce new values for the longitudinal parameters these are updated and a new score for the patient is calculated. In other words, the proposed method could be used to calculate the patient's risk every time the patient attends the office, whereas the traditional risk scales can be used with a longer time interval, as the prognosis is for 10 years. Thus, the two types of model could be used to assess the risk, for both the short term and the long term. Although discrepancies exist between shortterm and long-term predictions of CVD (Quispe et al., 2015), the regular use of short-term predictions, bearing in mind the variability of the risk factors, can complement the long-term cardiovascular models. In other words, our intention is for clinical practice to use the short-term model regularly in those patients who attend their physician's office frequently and use the longterm model in those who only attend occasionally. 
Obtaining simulations from longitudinal parameters is not easy and implies a

430

431

432

433

434

435

436

437

438

439

440

441

442

computational cost of about one minute with the statistical package $\mathrm{R}$ to implement a total of 100

using a normal computer. On the other hand, the historical values of the longitudinal parameters

are recorded in the clinical history, which nowadays is usually electronic (Palazón-Bru et al.,

2014). Given this situation, all the information needed to apply our models is already

computerised, so the algorithms implemented in the statistical package $\mathrm{R}$ can be adapted to the

underlying language of the database containing the values of the risk factors. Thus, all the

calculations will be immediate for the healthcare professional. In other words, just pressing a key

will be enough to bring up on the screen in a very short time the histogram shown in Fig. 2 and

Other S2, the theoretical points system and the set of values of the risk factors determining the median score. In addition, when the physician decides to intervene he or she will indicate the duration of the intervention and the possible values for the new patient. After introducing this new information the two histograms could be shown together (Figs. 2 and 3, and Other S2), which will enable the physician to see the benefit of the intervention.

As this algorithm was developed from a set of simulated data, we encourage others who have cardiovascular databases like that used here to implement a model with the characteristics described herein. Thus, if using real-life data achieves greater predictive precision we shall be able to apply this method to obtain the best short-term prognosis and thus take the most appropriate decisions for the benefit of the patient. Nevertheless, we should note that the method proposed is based on the combination of mathematical models already used in medicine; therefore in theory our model is quite correct as we have been extremely strict in each of the steps to follow. In practice we can determine the value of $\tilde{t}$ and the complexity of the models in 
451 order to apply the method proposed. Finally, and importantly, the algorithm developed in this

452 study can be used for other diseases or knowledge areas like the economy.

453

454 CONCLUSIONS

455 We developed an algorithm to construct cardiovascular risk scales based on a points system that

456 also takes into account the variability of the risk factors. These issues are important as the

457 popularity of points systems in clinical practice and the improved predictive accuracy using all

458 the information recorded in the clinical history will improve the currently used procedure. The

459 theoretical construction of our method is based on the combination of mathematical models

460 already used in medicine, taking into account the characteristics of each of these other models.

461 As mentioned, the prediction time and the structure of each of the models can change in practice,

462 as well as being used for other diseases apart from CVD or even applied to other areas of

463 knowledge. Finally, as we do not have real data available for its immediate application in clinical

464 practice, we encourage others to use our methods with their own data sets. In the case of CVD,

465 traditional cohort studies should be done, but recording repeated measurements of risk factors

466 both during the follow-up as well as for the period immediately prior to baseline

467

468 ACKNOWLEDGEMENTS

469 The authors thank Ian Johnstone for help with the English language version of the text. 
471

472

473

474

475

476

477

478

479

480

481

482

483

484

485

486

487 488

489

490

491

\section{REFERENCES}

American Diabetes Association. 2014. Standards of medical care in diabetes--2014. Diabetes Care 37 Supp1 1:S14-80.

Andersen PK, Gill RD. 1982. Cox's Regression Model for Counting Processes: A Large Sample Study. Annals of Statistics 10: 1100-1120.

Artigao-Ródenas LM, Carbayo-Herencia JA, Palazón-Bru A, Divisón-Garrote JA, SanchisDomènech C, Vigo-Aguiar I, Gil-Guillén VF; on behalf of GEVA. 2015. Construction and validation of a 14-year cardiovascular risk score for use in the general population: the PURASGEVA chart. Medicine (Baltimore) 94: e1980.

Brown ER, Ibrahim JG, DeGruttola V. 2005. A flexible B-spline model for multiple longitudinal biomarkers and survival. Biometrics 61:64-73.

Conroy RM, Pyörälä K, Fitzgerald AP, Sans S, Menotti A, De Backer G, De Bacquer D, Ducimetière P, Jousilahti P, Keil U, Njølstad I, Oganov RG, Thomsen T, Tunstall-Pedoe H, Tverdal A, Wedel H, Whincup P, Wilhelmsen L, Graham IM; SCORE project group. 2003. Estimation of ten-year risk of fatal cardiovascular disease in Europe: the SCORE project. European Heart Journal 24: 987-1003. 
492 Cooney MT, Dudina AL, Graham IM. 2009. Value and limitations of existing scores for the 493 assessment of cardiovascular risk: a review for clinicians. Journal of the American College of 494 Cardiology 54: 1209-1227.

495

496

Henderson R, Diggle P, Dobson A. 2000. Joint modelling of longitudinal measurements and 497 event time data. Biostatistics 1:465-480.

498

499

Hosmer DW, Lemeshow S. 2000. Applied Logistic Regression. New York: Wiley.

500

501

Hosmer DW, Lemeshow S, May S. 2008. Applied Survival Analysis: Regression Modeling of 502 Time-to-Event Data. New York: Wiley.

503

504

505

James PA, Oparil S, Carter BL, Cushman WC, Dennison-Himmelfarb C, Handler J, Lackland 506

DT, LeFevre ML, MacKenzie TD, Ogedegbe O, Smith SC Jr, Svetkey LP, Taler SJ, Townsend 506

RR, Wright JT Jr, Narva AS, Ortiz E. 2014. 2014 evidence-based guideline for the management of high blood pressure in adults: report from the panel members appointed to the Eighth Joint National Committee (JNC 8). JAMA 311: 507-520. Erratum in: JAMA 311: 1809.

Molinero LM. 2003. Modelos de riesgo cardiovascular. Estudio de Framingham. Proyecto

511 SCORE. Available at http://www.seh-lelha.org/pdf/modelries.pdf (accessed July 2015). 
513 National Cholesterol Education Program (NCEP) Expert Panel on Detection, Evaluation, and

514 Treatment of High Blood Cholesterol in Adults (Adult Treatment Panel III). 2002. Third Report

515 of the National Cholesterol Education Program (NCEP) Expert Panel on Detection, Evaluation,

516 and Treatment of High Blood Cholesterol in Adults (Adult Treatment Panel III) final report.

517 Circulation 106: 3143-3421.

518

519 Faucett CL, Thomas DC. 1996. Simultaneously modelling censored survival data and repeatedly

520 measured covariates: a Gibbs sampling approach. Statistics in Medicine 15:1663-1685.

521

522 National Heart, Lung, and Blood Institute (Boston University). 2015. The Framingham Heart

523 Study. Available at http://www.framinghamheartstudy.org/ (accessed July 2015).

524

525

526 directions. Circulation 121: 1768-1777.

527

528

Palazón-Bru A, Gil-Guillén VF, Orozco-Beltrán D, Pallarés-Carratalá V, Valls-Roca F, Sanchís-

Domenech C, Martín-Moreno JM, Redón J, Navarro-Pérez J, Fernández-Giménez A, Pérez-

Navarro AM, Trillo JL, Usó R, Ruiz E. 2014. Is the physician's behavior in dyslipidemia

531 diagnosis in accordance with guidelines? Cross-sectional ESCARVAL study. PLoS One 532 9:e91567. 
534 Perk J, De Backer G, Gohlke H, Graham I, Reiner Z, Verschuren M, Albus C, Benlian P, Boysen

535 G, Cifkova R, Deaton C, Ebrahim S, Fisher M, Germano G, Hobbs R, Hoes A, Karadeniz S,

536 Mezzani A, Prescott E, Ryden L, Scherer M, Syvänne M, Scholte op Reimer WJ, Vrints C,

537 Wood D, Zamorano JL, Zannad F; European Association for Cardiovascular Prevention \&

538 Rehabilitation (EACPR); ESC Committee for Practice Guidelines (CPG). 2012. European

539 Guidelines on cardiovascular disease prevention in clinical practice (version 2012). The Fifth

540 Joint Task Force of the European Society of Cardiology and Other Societies on Cardiovascular

541 Disease Prevention in Clinical Practice (constituted by representatives of nine societies and by

542 invited experts). European Heart Journal 33: 1635-1701. Erratum in: European Heart Journal

$543 \quad 33: 2126$.

545 Quispe R, Bazo-Alvarez JC, Burroughs Peña MS, Poterico JA, Gilman RH, Checkley W,

546 Bernabé-Ortiz A, Huffman MD, Miranda JJ; PERU MIGRANT Study; CRONICAS Cohort

547 Study Group. 2015. Distribution of Short-Term and Lifetime Predicted Risks of Cardiovascular

548 Diseases in Peruvian Adults. Journal of the American Heart Association 4:e02112.

Rizopoulos D. 2011. Dynamic predictions and prospective accuracy in joint models for longitudinal and time-to-event data. Biometrics 67: 819-829.

Rizopoulos D, Ghosh P. 2011. A Bayesian semiparametric multivariate joint model for multiple longitudinal outcomes and a time-to-event. Statistics in Medicine 30:1366-1380. 
556 Rizopoulos D. 2012. Joint Models for Longitudinal and Time-to-Event Data With Applications

557 in R. Boca Raton: CRC Press.

558

559 Stone NJ, Robinson JG, Lichtenstein AH, Bairey Merz CN, Blum CB, Eckel RH, Goldberg AC,

560 Gordon D, Levy D, Lloyd-Jones DM, McBride P, Schwartz JS, Shero ST, Smith SC Jr, Watson

561 K, Wilson PW; American College of Cardiology/American Heart Association Task Force on

562 Practice Guidelines. 2014. 2013 ACC/AHA guideline on the treatment of blood cholesterol to

563 reduce atherosclerotic cardiovascular risk in adults: a report of the American College of

564 Cardiology/American Heart Association Task Force on Practice Guidelines. Journal of the

565 American College of Cardiology 63(25 Pt B):2889-2934. Erratum in: Journal of the American

566 College of Cardiology 63(25 Pt B): 3024-3025.

567

Sullivan LM, Massaro JM, D'Agostino RB Sr. 2004. Presentation of multivariate data for clinical use: The Framingham Study risk score functions. Statistics in Medicine 23: 1631-1660. Review.

Verbeke G, Lesaffre E. 1997. The effect of misspecifying the random effects distribution in linear mixed models for longitudinal data. Computational Statistics and Data Analysis 23: 54156. longitudinal data and event times. Statistics in Medicine 25:143-163. 
578 Wang Y, Taylor JMG. 2001. Jointly Modeling Longitudinal and Event Time Data With

579 Application to Acquired Immunodeficiency Syndrome. Journal of the American Statistical 580 Association 96:895-905.

581

582 World Health Organization. 2014. The top 10 causes of death. Available at

583 http://www.who.int/mediacentre/factsheets/fs310/en/ (accessed July 2015).

585 Zeng D, Cai J. 2005. Simultaneous modelling of survival and longitudinal data with an 586 application to repeated quality of life measures. Lifetime Data Analysis 11:151-174. 


\section{Table $\mathbf{1}$ (on next page)}

Parameters ( $\square$ s) of the multivariate Cox regression model.

Abbreviations: SBP, systolic blood pressure; HbAlc, glycated haemoglobin. Goodness-of-fit (likelihood ratio test): $\square \quad 2=912.3, p<0.001$. 


\begin{tabular}{|c|c|c|}
\hline Variable & $\beta$ & $\mathrm{p}$-value \\
\hline Age (baseline) (per 1 year) & 0.0846 & $<0.001$ \\
\hline SBP (per 1 mmHg) & 0.00874 & $<0.001$ \\
\hline HbA1c (per 1\%) & 0.188 & $<0.001$ \\
\hline Atherogenic index (per 1 unit) & 0.191 & $<0.001$ \\
\hline Male gender & 0.479 & 0.001 \\
\hline Smoker (baseline) & 0.721 & $<0.001$ \\
\hline
\end{tabular}

1 


\section{Table 2 (on next page)}

Parameters of the joint models with the longitudinal parameters studied.

Abbreviations: SBP, systolic blood pressure; HbAlc, glycated haemoglobin; N/A, not applicable. *: term eliminated due to convergence problems. The strategy to eliminate variables is to eliminate down from the most complex terms to the most simple terms. Goodness-of-fit: 1) SBP: $>$ 2=371,574.1, p<0.001; 2) HbAlc: $\square 2=210,881.1, p<0.001 ; 3$ ) Atherogenic index: $\square 2=121,118.0, p<0.001$. 


\begin{tabular}{|l|c|c|c|c|c|c|c|}
\hline Variable & $\begin{array}{c}\text { SBP } \\
(\mathrm{mmHg})\end{array}$ & $\begin{array}{c}\mathrm{p}- \\
\text { value }\end{array}$ & $\begin{array}{c}\text { HbA1c } \\
(\%)\end{array}$ & $\begin{array}{c}\mathrm{p}- \\
\text { value }\end{array}$ & $\begin{array}{c}\text { Atherogenic } \\
\text { index }\end{array}$ & $\begin{array}{c}\mathrm{p}- \\
\text { value }\end{array}$ \\
\hline \multicolumn{7}{|c|}{ Event process } \\
\hline Male gender & 0.428 & $<0.001$ & 0.475 & $<0.001$ & 0.446 & $<0.001$ \\
\hline Age (per 1 year) & 0.0837 & $<0.001$ & 0.0840 & $<0.001$ & 0.0833 & $<0.001$ \\
\hline Smoker & 0.731 & $<0.001$ & 0.757 & $<0.001$ & 0.775 & $<0.001$ \\
\hline Parameter (per 1 unit) & 0.0085 & $<0.001$ & 0.216 & $<0.001$ & 0.195 & $<0.001$ \\
\hline \multicolumn{7}{|c|}{ Longitudinal process: fixed effects } \\
\hline 1 & 133.557 & $<0.001$ & 6.158 & $<0.001$ & 4.602 & $<0.001$ \\
\hline $\mathrm{t}$ & 0.0046 & $<0.001$ & 0.0001 & $<0.001$ & 0.0001 & $<0.001$ \\
\hline \multicolumn{7}{|l|}{ Longitudinal process: random effects } \\
\hline 1 & 21.683 & N/A & 1.346 & N/A & 1.324 & N/A \\
\hline $\mathrm{t}$ & 0.0358 & N/A & $*$ & $*$ & 0.0013 & N/A \\
\hline Residual & 8.933 & N/A & 0.357 & N/A & 0.302 & N/A \\
\hline
\end{tabular}




\section{Table 3(on next page)}

History of the control parameters of the cardiovascular risk factors included in our points system.

Abbreviations: SBP, systolic blood pressure; HbAlc, glycated haemoglobin. Time has a negative value because it refers to the measurements taken before the baseline situation and this was defined as $\mathrm{t}=0$. 


\begin{tabular}{|c|c|c|c|}
\hline Time (days) & SBP (mmHg) & HbA1c (\%) & Atherogenic index \\
\hline-360 & 152 & 5.1 & 3.56 \\
\hline-330 & 135 & 5.3 & 3.23 \\
\hline-270 & 164 & 4.7 & 3.45 \\
\hline-180 & 153 & 4.4 & 4.12 \\
\hline-90 & 170 & 5.0 & 4.15 \\
\hline 0 & 145 & 4.9 & 5.17 \\
\hline
\end{tabular}


1

Scoring system to predict cardiovascular diseases within 2 years.

Abbreviations: SBP, systolic blood pressure; HbAlc, glycated haemoglobin; TC, total cholesterol; HDL-c, HDL cholesterol. 


\begin{tabular}{cc}
\hline Age (years) & Score \\
\hline $\mathbf{1 8 - 2 9}$ & 0 \\
$\mathbf{3 0 - 3 9}$ & 2 \\
$\mathbf{4 0 - 4 9}$ & 4 \\
$\mathbf{5 0 - 5 9}$ & 6 \\
$\mathbf{6 0 - 6 9}$ & 8 \\
$\mathbf{7 0 - 7 9}$ & 10 \\
$\mathbf{2 8 0}$ & 12 \\
\hline
\end{tabular}

\begin{tabular}{cc}
\hline SBP $(\mathrm{mmHg})$ & Score \\
\hline$<120$ & 0 \\
$120-179$ & 1 \\
$\geq 180$ & 2 \\
\hline
\end{tabular}

\begin{tabular}{cc}
\hline Smoker & Score \\
\hline No & 0 \\
Yes & 2 \\
\hline
\end{tabular}

\begin{tabular}{cc}
\hline Gender & Score \\
\hline Female & 0 \\
Male & 1 \\
\hline
\end{tabular}

\begin{tabular}{cc}
\hline TC/HDL-C & Score \\
\hline$<4$ & 0 \\
\hline $4-5.99$ & 1 \\
$6-6.99$ & 2 \\
$\geq 7$ & 3 \\
\hline
\end{tabular}

\begin{tabular}{cc}
\hline HbA1c (\%) & Score \\
\hline$<\mathbf{5 . 7}$ & 0 \\
$\mathbf{5 . 7 - 6 . 4}$ & 1 \\
$\geq \mathbf{6 . 5}$ & 2 \\
\hline
\end{tabular}

\begin{tabular}{|ccc|}
\hline Total score & Group & Risk \\
\hline $\mathbf{0 - 8}$ & Low & $<1 \%$ \\
\hline $\mathbf{9 - 1 2}$ & Medium & $1-4 \%$ \\
\hline $\mathbf{1 3 - 1 4}$ & High & $5-9 \%$ \\
\hline$\geq 15$ & Very high & $>10 \%$ \\
\hline
\end{tabular}


2

Comparison between the proportions (\%) of expected and observed events in each of the different risk groups.

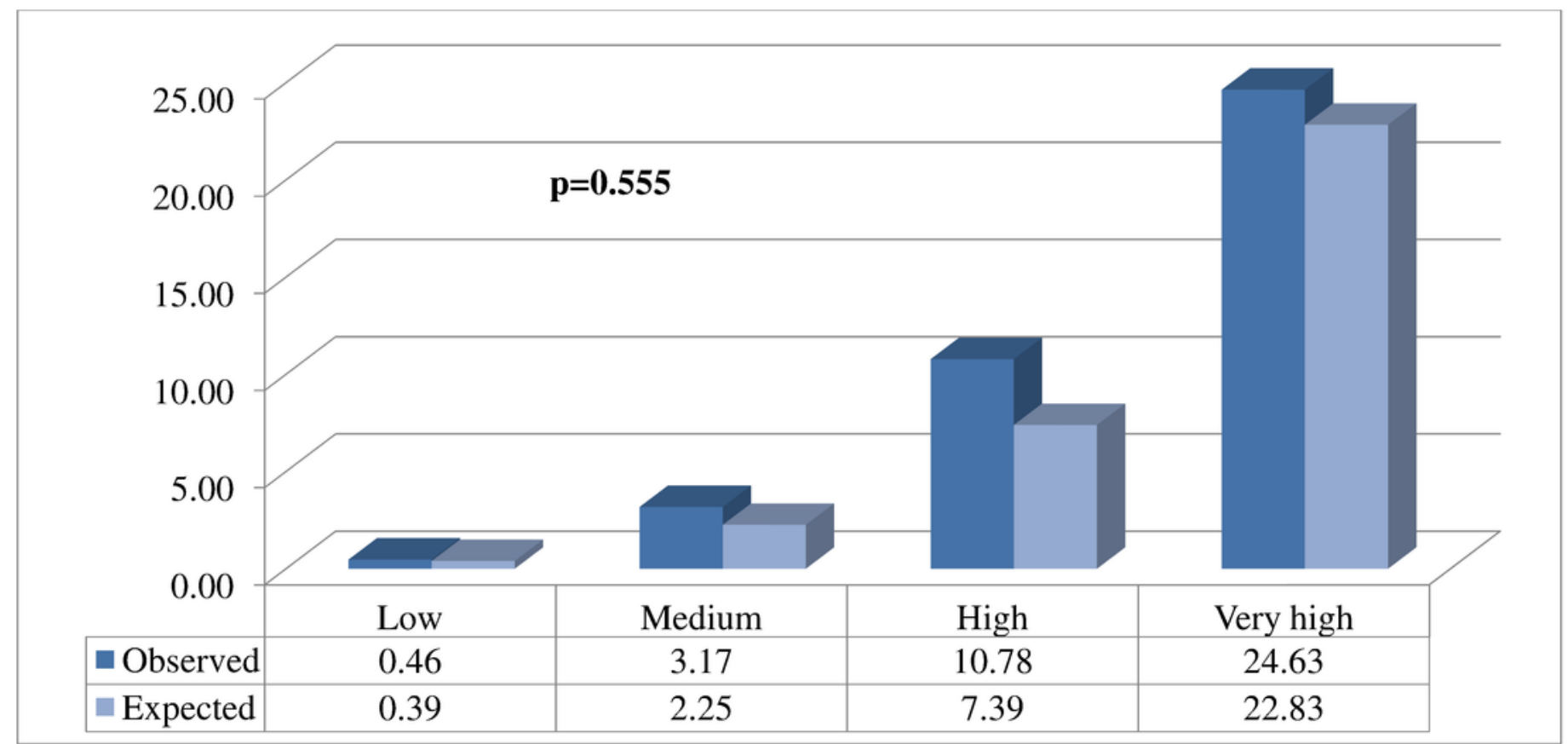


3

Cardiovascular risk of a theoretical new patient (pre-intervention).

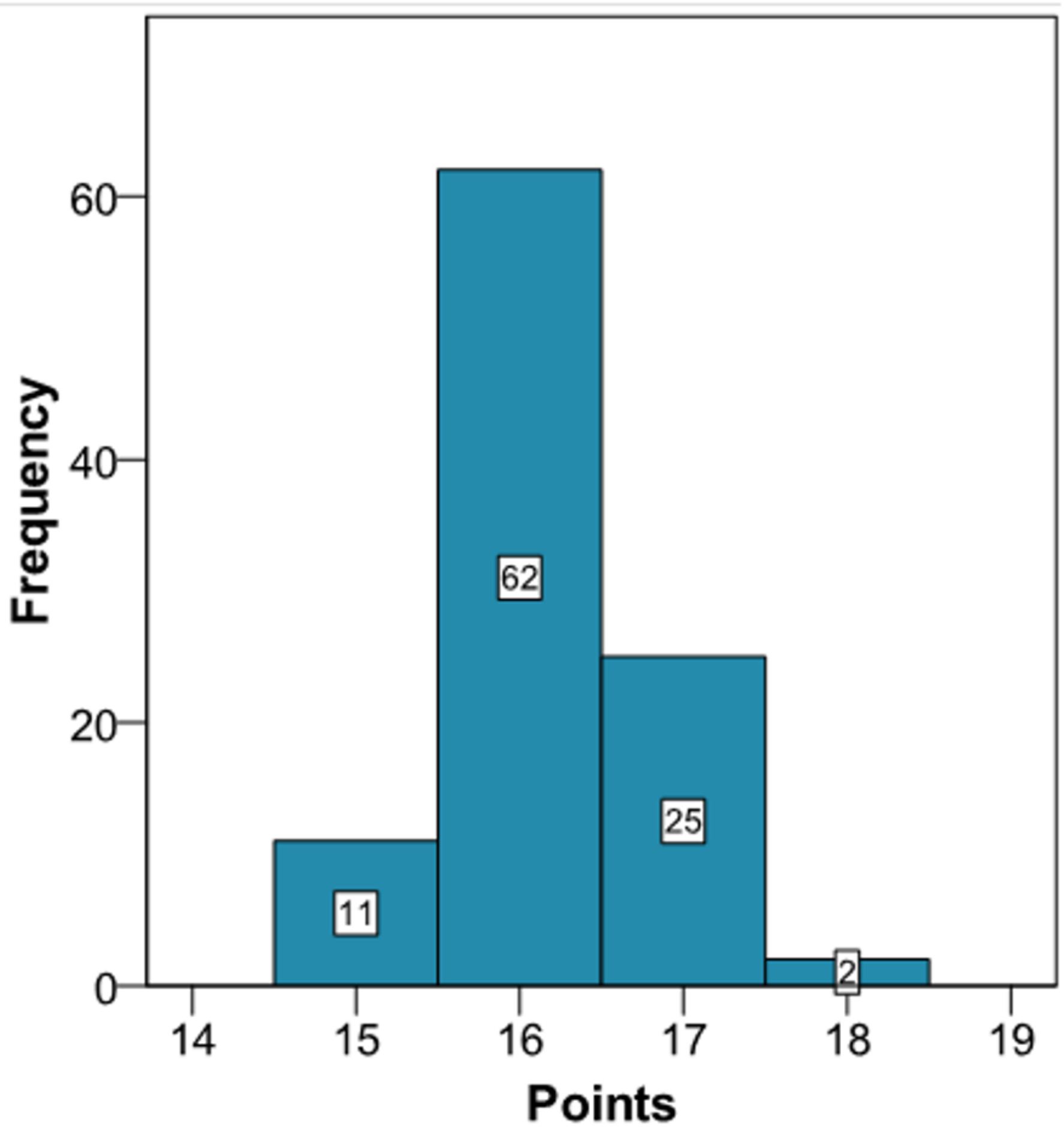


4

Cardiovascular risk of a theoretical new patient (post-intervention).

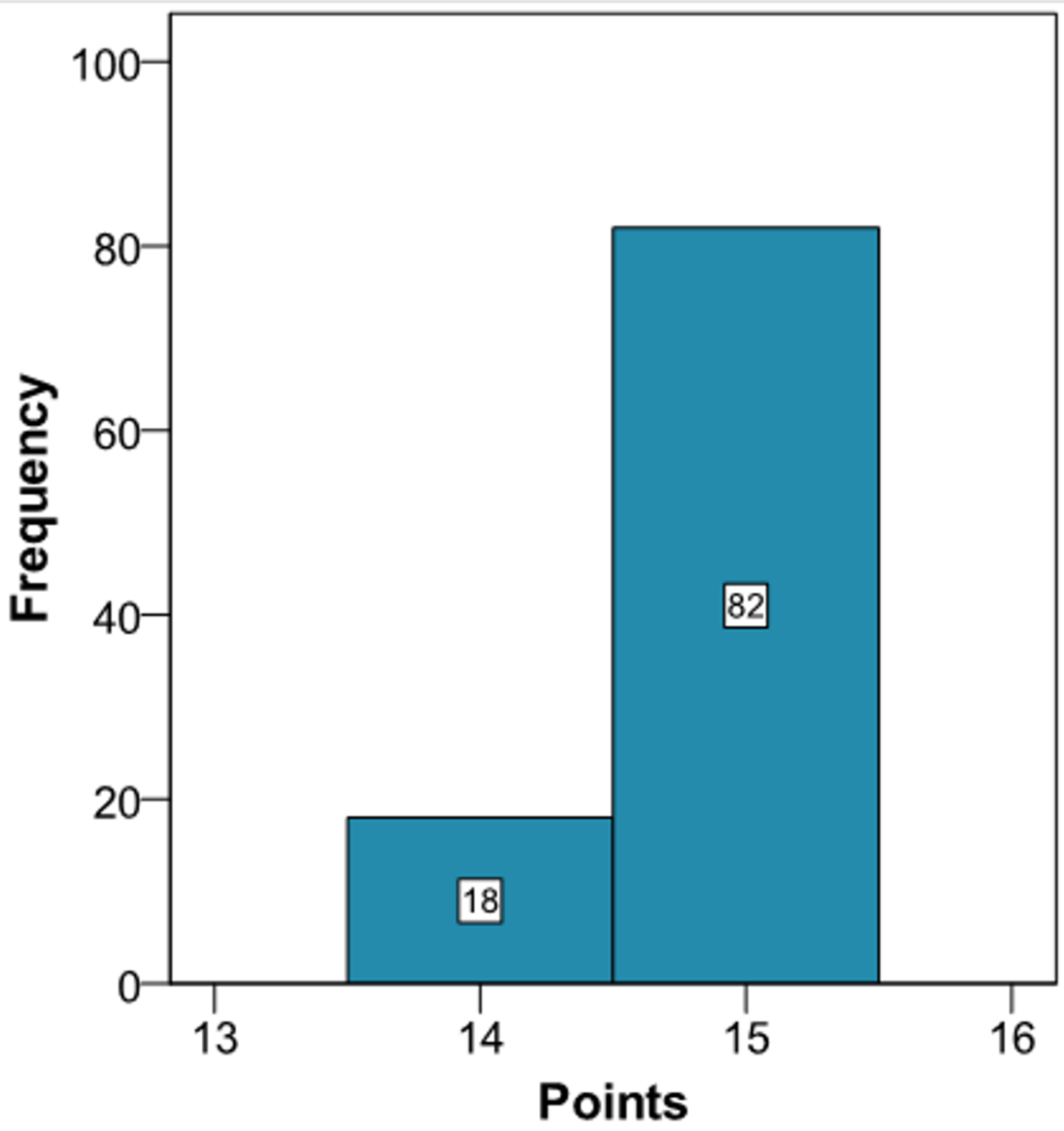

